\title{
Differences in the influences of ovariectomy and orchiectomy on the remodel- ing of mandibular condyle and femora in mice
}

Tadashi Fujta, Toshitsugu Kawata, Junji Ohtani, Masato Kaku, Chiyoko Tokimasa, Shinya Kohno, Keisuke Tsutsui, Kaoru Tenjo, Masahide Motokawa and Kazuo Tanne

Department of Orthodontics, Hiroshima University Faculty of Dentistry, Hiroshima, Japan

(Received 1 November 2001; and accepted 25 December 2001)

\begin{abstract}
Ovariectomy (OVX) and orchiectomy (ORX) are well known to increase bone turnover, however, the osteogenic effects are not fully understood. In this study, influences of sex hormone secretion cessation was examined for both sexes and different bones in terms of the change in the number of osteoblasts regarded as an index of bone formation. Male and female mice underwent ORX and OVX at 8 weeks old, respectively. In each of the experimental and control groups, mice were sacrificed 4 weeks after the surgery for OVX and ORX. The experimental mice exhibited an increase in the number of the osteoblasts in the condylar head, significantly different from the controls. Furthermore, in the experiment groups, the number of osteoblasts exhibited a more remarkable increase in the condyle than in the femora. This finding suggests that bone formation is accelerated by the reduction of sex hormone levels and the decrease in the trabecular bone volume is different between bones according to the degree of the enhanced bone formation or osteogenic activity.
\end{abstract}

Ovariectomy (OVX) and orchiectomy (ORX) are well known to increase bone turnover $(2,6,7,10$, $14)$, however, the influences on condylar remodeling are not fully understood. We previously examined the number of osteoclasts and the trabecular bone volume in the condylar head and the femora of OVX and ORX mice, indicating that the influences of OVX and ORX on bone remodeling vary by the sexual difference and the type of bones (4). On the other hand, it has been described that OVX promotes not only bone resorption but also bone formation, and the trabecular bone volume decreases because bone resorption exceeds bone formation ( 3 , $11,13)$.

According to these previous reports, it would be a reasonable assumption that the decreasing rate of trabecular bone volume may be varied due to different

Correspondence to: Dr. Tadashi Fujita, Department of Orthodontics, Hiroshima University Faculty of Dentistry, 12-3 Kasumi, Minami-ku, Hiroshima 734-8553, Japan

Tel: 082-257-5686; Fax: 082-257-5687

E-mail: seven@hiroshima-u.ac.jp bone formation rates between the condylar head and the femora, as is the case of bone resorption. The present study was thus designed to examine the influence of sex hormone secretion cessation on bone formation and the differences in both sexes and different bones in terms of changes in the number of osteoblasts as an index of bone formation.

Twenty C57BL/6J mice were housed in a breeding room (room temperature, $24 \pm 4$ degrees; relative humidity; $50 \pm 5 \%$ ), and fed a solid diet (CE2: Clea Japan, Tokyo, Japan). Under a general anesthesia with sodium pentobarbital, five male and female mice underwent ORX and OVX 8 weeks after birth, respectively. In each of the experimental and control groups, five mice were sacrificed 4 weeks after surgery for OVX and ORX. The body weight was measured every four days.

The mandibular condyles and femora were fixed with $4 \%$ paraformaldehyde, decalcified in EDTA ( $\mathrm{pH} 7.4$ ) for two weeks, and dehydrated in an ascending ethanol series $(70,80,90,95,99,100 \%)$. The specimens were embedded in paraffin and cut into frontal sections of $7 \mu \mathrm{m}$ thickness. The sections 
were stained with hematoxylin-eosin (H-E), azocarmine-aniline blue (AZAN) and tartrate-resistant acid phosphatase (TRAP) and observed microscopically. The sections stained with $\mathrm{H}-\mathrm{E}$ and AZAN were used for the histomorphometric analyses to count the number of osteoblasts in the condylar head and femora. The TRAP stained sections were used to count the number of osteoclasts in the condylar heads and femora. Bone histomorphometric analyses were performed in the subchondral area of the condyle and in an area of 1.0 $\mathrm{mm}^{2}$ within the secondary spongiosa of femur 1.0 $\mathrm{mm}$ below the epiphyseal growth plate along the central axis, using an image analysis program of NIH Image 1.59 (National Institutes of Health, Bethesda, MD, USA). Analysis of variances (ANOVA) and pairwise comparisons (Fisher) were performed to examine the differences between groups.

No remarkable differences in body weight were found between the OVX and ORX mice and the corresponding controls (Fig. 1). Thus, the experimental mice exhibited almost similar growth to the controls, indicating negligible influences of the surgery on general growth in terms of the changes in body weight.

The condylar heads of the OVX and ORX mice exhibited a significantly greater number of osteoblasts than those of the controls (Figs. 2, 3). For the femora, the OVX and ORX mice showed the same tendency as in the condylar head (Fig. 4). The rate of increase in osteoblast number was greater in the condylar head (3-fold increase) than in the femora (1.6-fold increase). However, no remarkable differences in the number of the osteoblasts were found between the OVX and ORX mice.

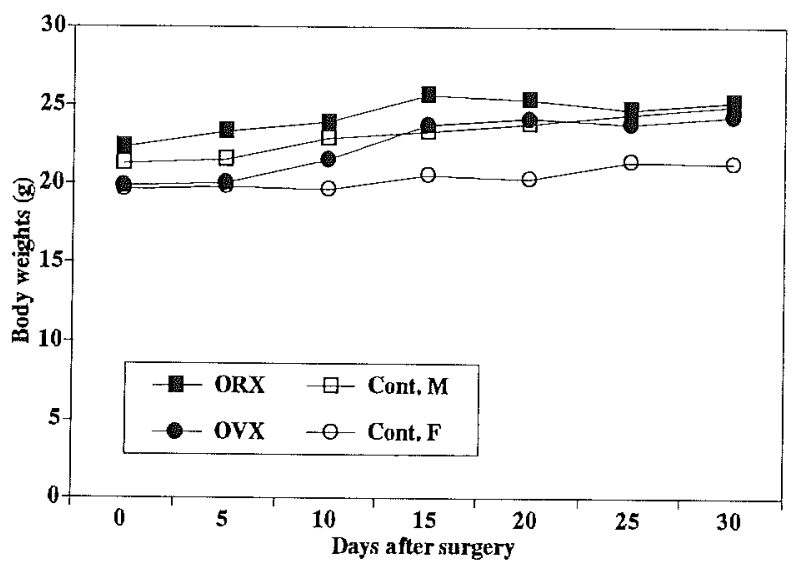

Fig. 1 Changes in body weights in the experimental (OVX, ORX) and control groups. $n=20$.
The trabecular bone volume of the condylar head decreased after OVX and ORX in accordance with the increase in the number of osteoclasts (Figs. 5, 6 ). In the femora, the trabecular bone volume showed the same tendency as in the condylar head, although the rate was greater than in the condylar head (Figs. 7, 8).

Decrease in the trabecular bone volume due to the lack of androgen and estrogen originates in more excessive bone resorption than bone formation. Influence of the sex hormone secretion cessation should be examined in both aspects of bone resorption and

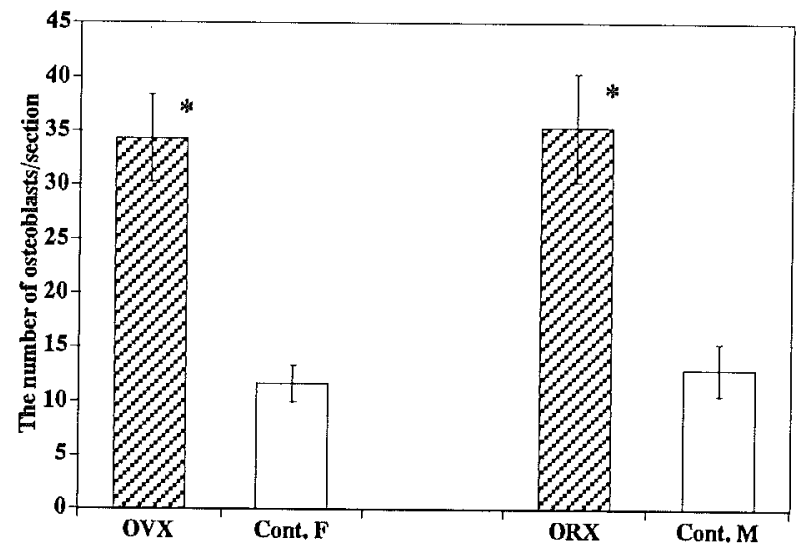

Fig. 2 The number of osteoblasts/section of the condylar head in the experimental (OVX, ORX) and control groups. ${ }^{*} P<0.05$ vs. control mice. $\mathrm{n}=20$.
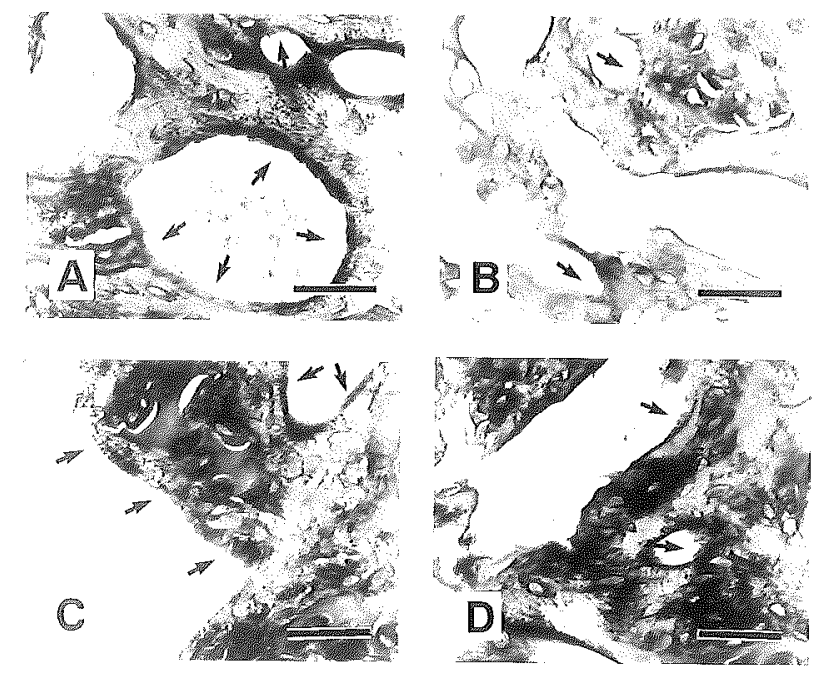

Fig. 3 Photomicrographs of the condyles in the OVX , ORX and control mice at 4 weeks after surgery. Arrows indicate osteoblasts.

A: OVX, B: Control (F), C: ORX, D: Control (M)

Bars denote $50 \mu \mathrm{m}$; AZAN stain; $\times 400$. 


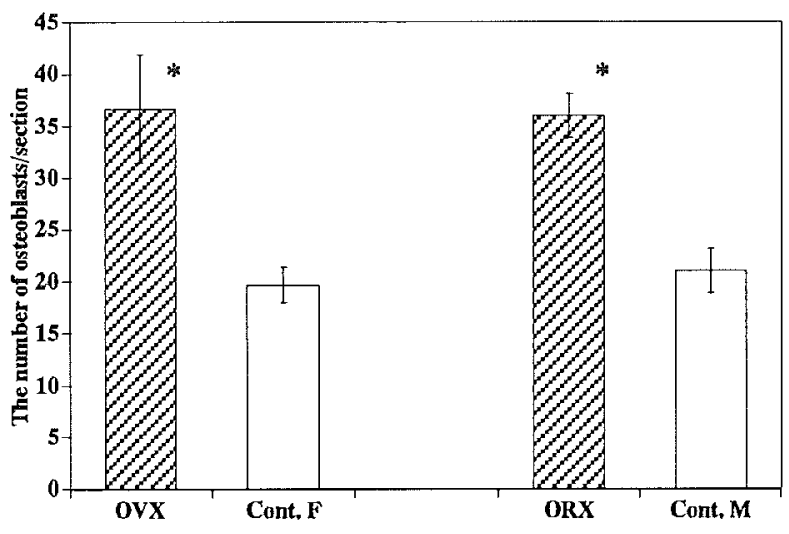

Fig. 4 The number of osteoblasts/section of the femora in the experimental (OVX, ORX) and control groups. $* P<0.05$ vs. control mice. $\mathrm{n}=20$.

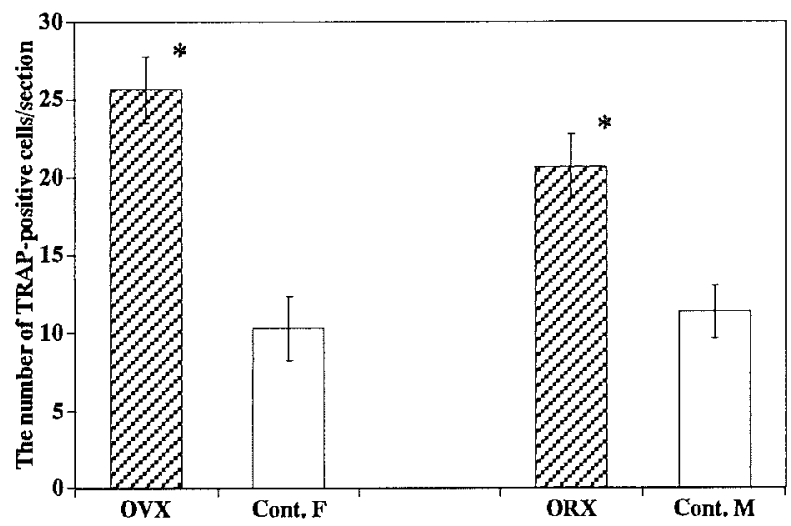

Fig. 5 The number of TRAP-positive cells/section of the condylar head in the experimental (OVX, ORX) and control groups. ${ }^{*} P<0.05$ vs. control mice. $\mathrm{n}=20$.

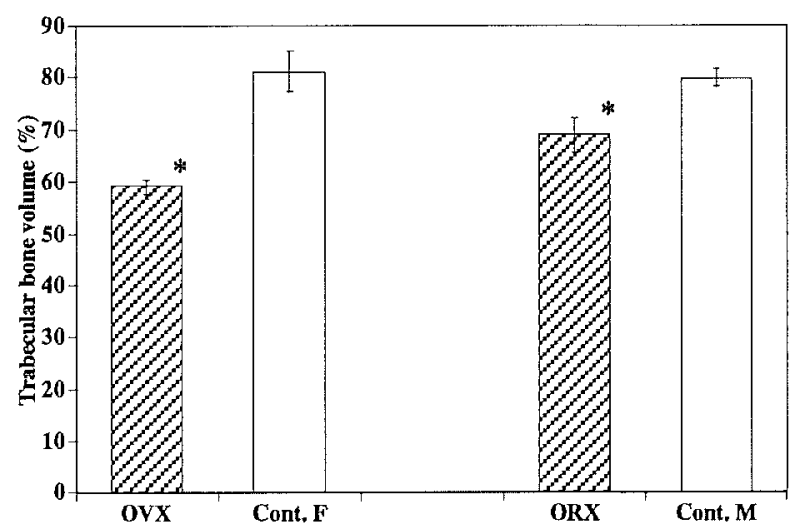

Fig. 6 The trabecular bone volume of the condylar head in the experimental (OVX, ORX) and control groups. ${ }^{*} P<0.05$ vs. control mice. $\mathrm{n}=20$.

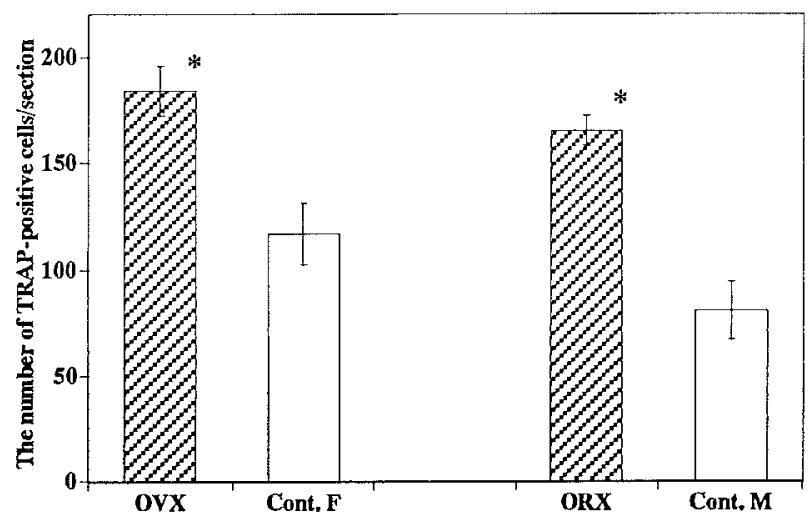

Fig. 7 The number of TRAP-positive cells/section of the femora in the experimental (OVX, ORX) and control groups. ${ }^{*} P<0.05$ vs. control mice, $\mathrm{n}=20$.

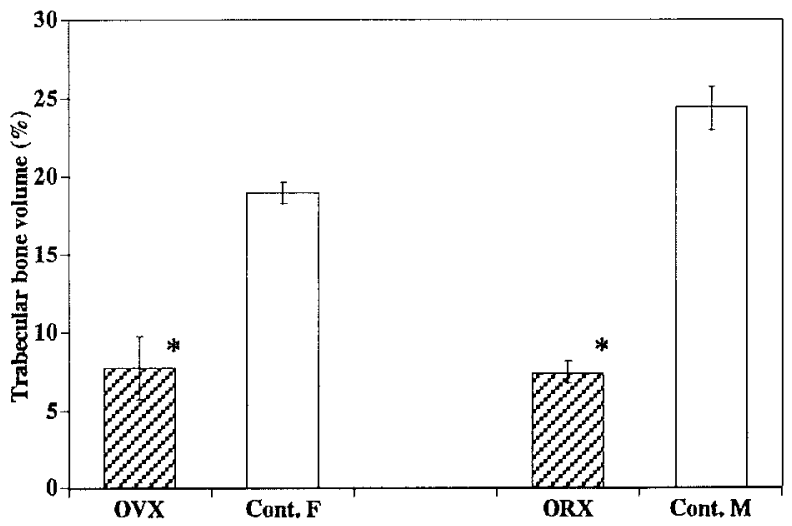

Fig. 8 The trabecular bone volume of the femora in the experimental (OVX, ORX) and control groups. $* P<0.05$ vs. control mice. $\mathrm{n}=20$.

\section{formation.}

It has been demonstrated that the estrogen and androgen receptors are expressed in the osteoblasts (1, 2 , 6). Since estrogen promotes the production of multiplication factors for bone formation such as insulin-like growth factor-1 (IGF-1) and transforming growth factor- $\beta$ (TGF- $\beta)(6)$, the increase in the trabecular bone volume by the estrogen may possibly be brought by the multiplication factors. However, the function of estrogen on osteoblast in vivo remains unclear. Wronski et al. reported that bone formation was found to be elevated maximally in OVX rats during the first month postovariectomy and increased bone turnover occurs during the early stages of estrogen deficiency. Furthermore they reported that osteoblasts were more difficult to identify in the older animals (12). This study suggested a 
possibility that bone formation was promoted by the lack of estrogen and androgen, if an impediment of bone formation exists. It is thus anticipated in future studies to elucidate a unknown factor involved in decrease in the trabecular bone volume.

Decrease rate in the trabecular bone volume was different between the condylar head and the femora. The difference may be due to the difference in the amount of bone formation. Another reason may be because of different internal structure of the bones and an interaction of mechanical stress. For the internal structure, it is pointed out that the amount of the spongiosa and the cortical bones is different between bones. In a biomechanical aspect, it is suggested that mechanical loading around the bone unit area is different from bone to bone.

Wronski et al. reported, on the basis of cellular parameters, that bone formation increased to a greater extent than bone resorption in tibiae of OVX rats, i.e. eight-fold increase in osteoblasts and two-fold increase in osteoclasts (12), although osteoclastic activity was significantly greater than osteoblastic activity in postmenopausal patients (5). It may be assumed, therefore, that the activities of osteoblasts and osteoclasts are different between the condylar head and femora. Furthermore, an interaction between mechanical stress and bone remodeling may be considered as an important factor to account for the present findings. Skeletal unloading causes inhibition of bone formation and the subsequent trabecular osteoporosis $(8,9)$. The condylar head is subjected to continuous mechanical stimuli from mastication, therefore, the decrease in trabecular bone volume might be limited to a smaller extent. Response to mechanical stimuli may be different between two bones. These assumptions established from the present findings are hopefully anticipated to be verified in future studies.

\section{REFERENCES}

1. Colvard D. S., Eriksen E. F., Keeting P. E., Wilson E. M., Lu- bahn D. B., French F. S., Riggs B. L. and Spelsberg T. C. (1989) Identification of androgen receptors in normal human osteoblast-like cells. Proc. Natl. Acad. Sci. U. S. A. 86, 854857.

2. Eriksen E. F., Colvard D. S., Berg N. J., Graham M. L., Mann K. G., Spelsberg T. C. and Riggs B. L. (1988) Evidence of estrogen receptors in normal human osteoblast-like cells. Science 241, 84-86.

3. Ernst M., Schmid C. and Froesch E. R. (1988) Enhanced osteoblast proliferation and collagen gene expression by estradiol. Proc. Natl. Acad. Sci. U. S. A. 85, 2307-2310.

4. Fujita T., Kawata T., Tokimasa C., Kaku M., Kawasoko S. and Tanne K. (1998) Influences of ovariectomy and orchiectomy on the remodeling of mandibular condyle in mice. $J$. Craniofac. Genet. Dev. Biol. 18, 164-170.

5. Gruber H. E., Ivey J. L., Thompson E. R., Chesnut C. H. 3D. and Baylink D. J. (1986) Osteoblast and osteoclast cell number and cell activity in postmenopausal osteoporosis. Miner: Electrolyte. Metab. 12, 246-254.

6. Komm B. S., Terpening C. M., Benz D. J., Graeme K. A., Gallegos A., Korc M., Greene G. L., O'malley B. W. and Haussler M. R. (1988) Estrogen binding, receptor mRNA, and biologic response in osteoblast-like osteosarcoma cells. Science 241, 81-84.

7. Lindgren J. U. and Lindholm T. S. (1979) Effect of 1-alphahydroxyvitamin D3 on osteoporosis in rats induced by oophorectomy. Calcif. Tissue Int. 27, 161-164.

8. Machwate M., Zerath E., Holy X., Hott M., Modrowski D., Malouvier A. and Marie P. J. (1993) Skeletal unloading in rat decreases proliferation of rat bone and marrow-derived osteoblastic cells, Am. J. Physiol. 264, E790-799.

9. Machwate M., Zerath E., Holy X., Pastoureau P. and Marie P. J. (1994) Insulin-like growth factor-I increases trabecular bone formation and osteoblastic cell proliferation in unloaded rats. Endocrinology 134, 1031-1038.

10. Saville P. D. (1969) Changes in skeletal mass and fragility with castration in the rat; a model of osteoporosis. $J$. Am. Geriatr: Soc. 17, 155-166.

11. Takano-Yamamoto T. and Rodan G. A. (1990) Direct effects of 17-estradiol on trabecular bone in ovariectomized rats. Proc. Natl. Acad. Sci. U. S. A. 87, 2172-2176.

12. Wronski T. J., Cintron M. and Dann L. M. (1988) Temporal relationship between bone loss and increased bone turnover in ovariectomized rats. Calcif. Tissue Int. 43, 179-183.

13. Wronski T. J., Lowry P. L., Walsh C. C. and Ignaszewski L. A. (1985) Skeletal alterations in ovariectomized rats. Calcif. Tissue Int. 37, 324-328.

14. Wronski T. J., Schenck P. A., Cintron M. and Walsh C. C. (1987) Effect of body weight on osteopenia in ovariectomized rats. Calcif. Tissue Int. 40, 155-159. 